\title{
Cluster units in export garment industry: SWOT Analysis
}

\author{
${ }^{1}$ Mrs.K.S.Alamelu mangai sudha, ${ }^{2}$ Dr.Rameshwari Ramchandra \\ ${ }^{I}$ Tiruppur kumaran college for women Tirupur \\ ${ }^{2}$ PSGR Krishnammal college for women Coimbatore
}

\section{Introduction}

A SWOT analysis covers four areas of consideration. The first "strengths" takes into account internal attributes that will positively affect the business objective. The second area, "weaknesses," identifies potential weak-links within the company's control that could contribute to a detrimental outcome. The third category, "opportunities," analyzes external conditions that favor end-goal achievement. Finally, external "threats" that are beyond the company's control face evaluation.

\subsection{INDIAN GARMENT INDUSTRY}

Garment industry is very important for a country, in terms of trade, GDP and employment and has contributed significantly to the economy in several countries. Garment industry provide opportunities for export diversification and expansion of manufactured exports for many countries. Garment industry plays a very important role in Indian economy. India is the world's second largest producer of textiles and garments after China. It has made significant progress over the years not only in respect of its contributions to industrial production, exports and generation of employment but also in achieving a high degree of sophistication, quality up gradation, cost reduction and standardisation capable of withstanding stiff international competition. India is now the strategic choice, with a vertically integrated, flexible and comprehensive production infrastructure. Skilled labour, production-friendly systems and stable economic environment helped India to deliver wide varieties and huge volumes for the global market. The Vision Statement for the textiles industry for the 11th Five Year Plan (2007-12) sees India securing a 7 percent share in the global textiles trade by 2012. At current prices, the Indian textiles industry is valued at US\$ 55 billion, 64 per cent of which caters to domestic demand. Total textile exports during April-March 2010-11 stood at US\$ 12.5 billion as against US\$ 11.3 billion during the corresponding period of the previous year, according to the data released by DGCI\&S, Kolkata.The technical textiles segment is expected to grow by 11percent per annum till 2012-13 and is likely to grow at 6-8 per cent per annum till 2020 without any policy interventions. If the government intervenes by way of regulatory push, the growth of technical textiles industry can be estimated at 12-15 per cent per annum till 2020, according to Rita Menon, Secretary, Union Ministry of Textiles. She added that the technical textiles segment in India has the potential to attract investment and create additional employment opportunities in coming years. She further said that investments of US\$ 1.1 billion are expected by 2012 and employment is expected to increase to 1.2 million by 2012 .

\subsection{TIRUPUR GARMENT INDUSTRY}

Tirupur has nearly 1500 exporting firms, which symbolises the spirit of entrepreneurship in the country. Tirupur has emerged as a town, which is synonymous with knitwear, and export culture and is rated as the most important potential future growth by the leading global markets. Tirupur's performance in Textiles originates from its performance in technology and the quality of its macro economic environment. It also derives much of its ascent from improved perception of its public service. Buyers from 35 countries frequently visit Tirupur. Tirupur can deliver customized samples in less than 12 hours; half a million pieces in a matter of days. 56\% of India's total knitwear exports come from Tirupur. This has been recognized in the Export Import Policy, 2002-07 of Government of India which conferred the status of 'Town of Export Excellence' to Tirupur. The rich availability of Raw materials, being in close proximity to Coimbatore which is a major centre of cotton spinning industry in the Country makes Tirupur being able to access its basic raw materials quickly and as and when required.The strong entrepreneurial skills and personalized management contributes to efficient management of negotiations and direct control of operations causing cost effective competitiveness of the Industry; quick delivery and quality products add dimension to the Tirupur's prowess as a centre to outsource excellent products 


\subsection{CLUSTER}

Cluster is a term used by everyone in the industry to describe work (a 'job") shared by firms specializing in different processes, within firms between different units Specializing in different processes, or to refer to contract work organized through traders. These arrangements can be considered structurally, but they can also be considered as a social relation of production where by owners of firms are able avoid problems associated with the direct control of large labor forces. Thus:

* "out contracting" takes place between firms specializing in different processes and enables firms to make choices about the extent to which they vertically integrate parts of the production process

* "in contracting" has developed within some of the larger firms where an owner(s) employs a "job work contractor" for each unit. The contractor acts as a production manager who is also responsible for employing labor for a particular "job". This system again sets up process divisions, but this time within a firm( Cawthome, 1995:47).

The horizontal and vertical networking is complex and dense. Some patterns can be discerned. The entrepreneurs grouped into three categories: namely,exporter-manufacturers, non-exporter manufacturers and merchant exporters. However, they further suggest that:

(a) in each of these categories there are large, medium and small firms;

(b) the export-manufacturers and the merchant exporters who dominate the scene control (formally and informally)a variety of enterprises spanning the industry both Horizontally and vertically;

(c) the non-exporter manufacturer category includes both those producing for exporters

(and hence strictly speaking sub contracting units) as also those producing exclusively

for the domestic market.

The need to stick to delivery schedules and maintain internationally acceptable standards in quality, finish etc., has had its own impact on the manner in which production is organized. It has not led to all operations being brought under one roof. The large exporter-manufacturers and merchant exporters teamed up with several enterprises covering different segments of the production process. The mutual exchange of information and assistance provided( if necessary)between the members of the team (in technology, market on the one hand, and expertise relating to the different processes on the other) enables each of the segments to operate at internationally acceptable standards of production.

\subsection{TIRUPUR CLUSTER}

Tirupur can be easily classified as an industrial cluster that typifies an organic relationship between firms, both horizontally and vertically. This is because of the dense network of production organization that exists within the region. The production of garments in the cluster is segmented into separate modules and firms participate indifferent portions of the value chain. Everywhere in the small town one can easily notice how activities revolve around the production and sale of knitwear garments. There are a large number of suppliers selling different grades of yarn and these yarns are procured by producers to initiate the production process. Most of the garment producers or exporters generally confine to stages such as cutting, stitching and finishing activities and get the prior stages done by specialized firms. The first stage can be termed as fabrication or knitting. The knitted fabric is then sent to processing units which include operations such as mercerization, dyeing and compacting Printing is the next stage in the production process. This is done after the garments are cut according to specific designs by the exporting firm. In most of the cases printing job is outsourced but in some cases the exporting units do the printing job in house.the final stage is the CMT process comprises of cutting according to the pattern and there after stitching.

\section{Review Of Literature}

Survey of India's export potential of textile and made up garments conducted by economic and scientific research foundation (1969) points out the woeful share of India in global garment trade due to poor technology and marketing. The survey recommends for special scheme for improvement in those areas .Jaitley, 2003 has stated that India's export growth rate of $18 \%$, next to that of China with $22 \%$ was the second highest among the top 30 leading exporters in world merchandise trade in 2002.Veembur, 2004.has studied that from 1990 to 2003, the average growth rate of Indian textile and apparel production was $5 \%$. Furthermore, this figure has been predicted to be as high as $15 \%$ after 2005 .Cawthorne, (1995). "Tirupur is a textiles town par excellence. It lies in the heart of a cotton producing area. It has a long history as a processor of raw cotton, as a centre for handloom weaving and as a cotton trading centre. Its cotton exchange traditionally set the price of raw cotton in the state of Tamil Nadu. Moreover, 84 percents of factory industry in Tirupur is textile related.According to Swaminathan \& Jeyaranjan(1994), Tirupur's direct knitwear exports in 1993 were worth nearly US\$ 500 million; whereas if indirect exports are also included (taking note of exports of Tiruppur made garments sold through Bombay and Delhi based traders and producers), this figure jumps to over US\$900 million 


\section{Methodology}

Having identified the unique nature of the garment industry with regard to the variable decision making process and special significance in the Indian economy, the study focused on the direction and composition of garment industry. The heart of the garment industry belong to cluster which is the parallel production process. The undisputed advantage of India with regard to the plentiful availability of resources on the one side and the demanding quality and the depressing price on the other side called for a scientific enquiry in to the cluster units in Tirupur.The methodology was formulated keeping this in mind. SWOT analysis is conducted to identify the strengths, weaknesses, opportunities and threats before proceeding to the formulation of long and short term strategy. This article seeks to identify the SWOT variables through descriptive study, a structured interview schedule have been framed and 477 cluster units were surveyed by interview method in Tirupur, Henry Garett scale and multiple regression was adopted for analyzing the strength weakness, opportunity and threat factors

\section{Results And Discussions}

In Tirupur city, there are many cluster job works available, as there exists huge order for production, all heavy export oriented industries provide job works to the ancillary industries and try to complete the export order and earn enough foreign exchange the common job works which are available in Tirupur city was taken for the purpose of analysis viz., 1. Screen printing, 2. Dyeing,3.Knitting.

TABLE-1

Type of cluster unit under taken

\begin{tabular}{|c|c|c|c|}
\hline S. No & Work & No., of respondent & Percent \\
\hline 1 & Screen printing & 84 & 17.6 \\
\hline 2 & dyeing & 266 & 55.8 \\
\hline 3 & knitting & 127 & 26.6 \\
\hline & Total & 477 & 100.0 \\
\hline
\end{tabular}

Table 1 details out the type of cluster job work undertaken. From the analysis it has been understood that 55.8 percent of the respondents were involved in dyeing work, 26.6 percent of the respondent were involved in knitting works, and a remaining 17.6 percent of the respondents were involved in screen printing works.

TABLE-2

LEVEL OF AGREEMENT ON THE VARIOUS STRENGTH FACTORS OF THE PERFORMANCE OF CLUSTER UNITS

\begin{tabular}{|c|l|c|c|c|c|c|c|c|}
\hline S.No & $\begin{array}{c}\text { Strength } \\
\text { Factors }\end{array}$ & $\begin{array}{c}\text { Strongly } \\
\text { Agree }\end{array}$ & Agree & Neutral & Disagree & $\begin{array}{c}\text { Strongly } \\
\text { disagree }\end{array}$ & $\begin{array}{c}\text { Total } \\
\text { Score }\end{array}$ & $\begin{array}{c}\text { Mean } \\
\text { Rank }\end{array}$ \\
\hline 1 & Availability of & $\begin{array}{c}456 \\
(26.83)\end{array}$ & $\begin{array}{c}328 \\
(19.3)\end{array}$ & $\begin{array}{c}365 \\
(21.4)\end{array}$ & $\begin{array}{c}328 \\
(19.3)\end{array}$ & $\begin{array}{c}222 \\
(13.06)\end{array}$ & 1699 & V \\
\hline 2 & $\begin{array}{l}\text { Law material } \\
\text { material }\end{array}$ & $\begin{array}{c}652 \\
(34.6)\end{array}$ & $\begin{array}{c}320 \\
(16.9)\end{array}$ & $\begin{array}{c}219 \\
(11.6)\end{array}$ & $\begin{array}{c}365 \\
(19.29)\end{array}$ & $\begin{array}{c}328 \\
(17.40)\end{array}$ & 1884 & II \\
\hline 3 & $\begin{array}{l}\text { Labor cost } \\
\text { advantage }\end{array}$ & $\begin{array}{c}315 \\
(28.02)\end{array}$ & $\begin{array}{c}321 \\
(28.5)\end{array}$ & $\begin{array}{c}222 \\
(19.7)\end{array}$ & $\begin{array}{c}152 \\
(13.5)\end{array}$ & $\begin{array}{c}114 \\
(10.14)\end{array}$ & 1124 & X \\
\hline 4 & $\begin{array}{l}\text { Skilled labor } \\
\text { availability }\end{array}$ & $\begin{array}{c}480 \\
(32.78)\end{array}$ & $\begin{array}{c}365 \\
(24.93)\end{array}$ & $\begin{array}{c}312 \\
(21.3)\end{array}$ & $\begin{array}{c}216 \\
(14.7)\end{array}$ & $\begin{array}{c}91 \\
(6.21)\end{array}$ & 1464 & IX \\
\hline 5 & $\begin{array}{l}\text { Availability of } \\
\text { designers }\end{array}$ & $\begin{array}{c}526 \\
(32.85)\end{array}$ & $\begin{array}{c}425 \\
(26.5)\end{array}$ & $\begin{array}{c}326 \\
(20.3)\end{array}$ & $\begin{array}{c}231 \\
(14.4)\end{array}$ & $\begin{array}{c}93 \\
(5.8)\end{array}$ & 1601 & VIII \\
\hline 6 & $\begin{array}{l}\text { Smallness of } \\
\text { units }\end{array}$ & $\begin{array}{c}492 \\
(30.38)\end{array}$ & $\begin{array}{c}414 \\
(25.5)\end{array}$ & $\begin{array}{c}326 \\
(22.14)\end{array}$ & $\begin{array}{c}235 \\
(14.5)\end{array}$ & $\begin{array}{c}152 \\
(9.3)\end{array}$ & 1619 & VII \\
\hline 7 & $\begin{array}{l}\text { Ability to } \\
\text { supply in small } \\
\text { units or } \\
\text { quantity }\end{array}$ & $\begin{array}{c}456 \\
(27.88)\end{array}$ & $\begin{array}{c}432 \\
(26.14)\end{array}$ & $\begin{array}{c}362 \\
(22.7)\end{array}$ & $\begin{array}{c}154 \\
(9.4)\end{array}$ & $\begin{array}{c}231 \\
(14.12)\end{array}$ & 1635 & VI \\
\hline 8 & $\begin{array}{l}\text { Capability in } \\
\text { product } \\
\text { development/ } \\
\text { adaptation }\end{array}$ & $\begin{array}{c}426 \\
(29.09)\end{array}$ & $\begin{array}{c}354 \\
(24.1)\end{array}$ & $\begin{array}{c}333 \\
(22.7)\end{array}$ & $\begin{array}{c}136 \\
(9.2)\end{array}$ & $\begin{array}{c}215 \\
(14.6)\end{array}$ & 1464 & IX \\
\hline 9 & $\begin{array}{l}\text { Availability of } \\
\text { Infrastructure }\end{array}$ & $\begin{array}{c}546 \\
(29.80)\end{array}$ & $\begin{array}{c}435 \\
(23.7)\end{array}$ & $\begin{array}{c}514 \\
(28.0)\end{array}$ & $\begin{array}{c}115 \\
(6.2)\end{array}$ & $\begin{array}{c}222 \\
(12.11)\end{array}$ & 1832 & III \\
\hline 10 & Favorable & 544 & 435 & 432 & 126 & 154 & 1691 & IV \\
\hline
\end{tabular}


Cluster units in export garment industry: SWOT Analysis

\begin{tabular}{|c|l|c|c|c|c|c|c|c|}
\hline & $\begin{array}{l}\text { institutional } \\
\text { frame work }\end{array}$ & $(32.17)$ & $(25.7)$ & $(25.5)$ & $(7.4)$ & $(9.1)$ & & \\
\hline 11 & Incentives & $\begin{array}{c}632 \\
(39.56)\end{array}$ & $\begin{array}{c}521 \\
(25.1)\end{array}$ & $\begin{array}{c}542 \\
(26.2)\end{array}$ & $\begin{array}{c}225 \\
(10.88)\end{array}$ & $\begin{array}{c}148 \\
(7.15)\end{array}$ & 2068 & I \\
\hline
\end{tabular}

From the above table it could be understood that of the various factors taken for study, The I rank was secured by the factor X11- Incentives with a total score of 2068, The II and III ranks were secured by the factors of X2- Low cost of raw material, X9 - Availability of infrastructure with a total score of 1884, 1832 respectively. The IV, V, VI,VII ranks were secured by the factors of X10- Favorable institutional frame work, X1Availability of raw material, X7- Ability to supply in small units or quantity, X6-smallness of units with a total score of 1691, 1699, 1635,1619 respectively. The VIII , IX, X,X1 ranks were secured by the factors of X5Availability of designers, X8- Capability in product development/adoption, X4- skilled labour force availability X3- Labour cost advantage with a total score of $1601,1464,1124$ respectively.

\section{SWOT Analysis - Strength}

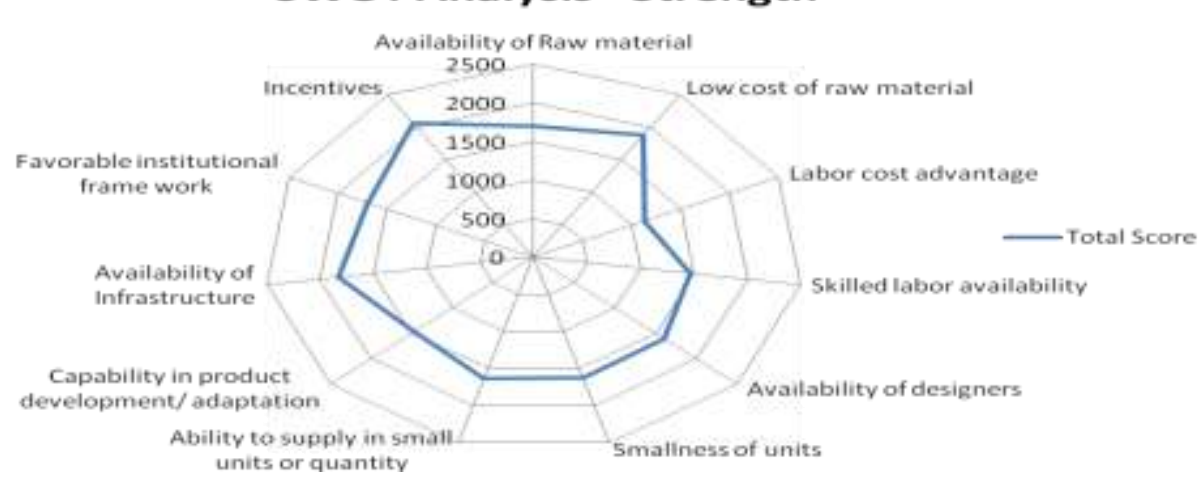

Model Summary

\begin{tabular}{|c|c|c|c|c|}
\hline Model & $\mathbf{R}$ & R Square & Adjusted R Square & $\begin{array}{l}\text { Std. Error of the } \\
\text { Estimate }\end{array}$ \\
\hline 1 & .820 (a) & .673 & .665 & .77362 \\
\hline
\end{tabular}

a Predictors: (Constant), incentives, skilled labour availability, capability in product development and or adaption, historical back ground of the location \& availability of allied industry has facilitated the growth, availability of support services such as banks have facilitated the growth, labour cost advantage, smallness of units, ability to supply in small units or quantity, availability of conductive climate has facilitated the growth, favorable institutional frame work, availability of water facility has facilitated the growth

\begin{tabular}{|l|l|r|r|r|r|c|}
\hline Model & & $\begin{array}{c}\text { Sum of } \\
\text { Squares }\end{array}$ & \multicolumn{1}{c|}{ Df } & Mean Square & \multicolumn{1}{c|}{ F } & \multicolumn{1}{c|}{ Sig. } \\
\hline 1 & Regression & 571.528 & 11 & 51.957 & 86.813 & $.000(\mathrm{a})$ \\
\hline & Residual & 278.300 & 465 & .598 & & \\
\hline & Total & 849.828 & 476 & & & \\
\hline
\end{tabular}

a Predictors: (Constant), incentives, skilled labour availability, capability in product development and or adaption, historical back ground of the location \& availability of allied industry has facilitated the growth, availability of support services such as banks have facilitated the growth, labour cost advantage, smallness of units, ability to supply in small units or quantity, availability of conductive climate has facilitated the growth, favorable institutional frame work, availability of water facility has facilitated the growth b Dependent Variable: type of cluster job work under taken 
Cluster units in export garment industry: SWOT Analysis

\begin{tabular}{|c|c|c|c|c|c|c|}
\hline \multicolumn{7}{|c|}{ Coefficients(a) } \\
\hline \multirow{2}{*}{ Model } & & \multicolumn{2}{|c|}{$\begin{array}{c}\text { Unstandardized } \\
\text { Coefficients }\end{array}$} & \multirow{2}{*}{$\begin{array}{c}\begin{array}{c}\text { Standardized } \\
\text { Coefficients }\end{array} \\
\text { Beta }\end{array}$} & \multirow{2}{*}{$\mathbf{t}$} & \multirow{2}{*}{ Sig. } \\
\hline & & B & $\begin{array}{l}\text { Std. } \\
\text { Error }\end{array}$ & & & \\
\hline \multirow[t]{12}{*}{1} & (Constant) & .577 & .084 & & 6.907 & .000 \\
\hline & $\begin{array}{l}\text { availability of water facility } \\
\text { has facilitated the growth }\end{array}$ & -.798 & .880 & -.800 & -.906 & .365 \\
\hline & $\begin{array}{l}\text { availability of conductive } \\
\text { climate has facilitated the } \\
\text { growth }\end{array}$ & .319 & .351 & .321 & .908 & .365 \\
\hline & $\begin{array}{l}\text { historical back ground of the } \\
\text { location \& availability of } \\
\text { allied industry has facilitated } \\
\text { the growth }\end{array}$ & .536 & .184 & .537 & 2.915 & .004 \\
\hline & $\begin{array}{l}\text { availability of support } \\
\text { services such as banks have } \\
\text { facilitated the growth }\end{array}$ & -.165 & .205 & -.166 & -.802 & .423 \\
\hline & labour cost advantage & .696 & .299 & .702 & 2.328 & .020 \\
\hline & skilled labour availability & -.036 & .186 & -.036 & -.194 & .846 \\
\hline & smallness of units & 1.075 & .294 & 1.070 & 3.655 & .000 \\
\hline & $\begin{array}{l}\text { ability to supply in small } \\
\text { units or quantity }\end{array}$ & -.116 & .321 & -.116 & -.362 & .718 \\
\hline & $\begin{array}{l}\text { capability in product } \\
\text { development and or adaption }\end{array}$ & .107 & .207 & .107 & .515 & .607 \\
\hline & $\begin{array}{l}\text { favorable institutional frame } \\
\text { work }\end{array}$ & .998 & .390 & .994 & 2.562 & .011 \\
\hline & incentives & -1.796 & .974 & -1.797 & -1.844 & .066 \\
\hline
\end{tabular}

a Dependent Variable: type of cluster job work under taken

When the multiple regression technique was adopted it could be understood that all the variables which are taken in to account for analyzing the strength factors shows a positive significance for those cluster units who has taken different job works

TABLE-3

LEVEL OF AGREEMENT ON THE VARIOUS WEAKNESS FACTORS OF THE PERFORMANCE OF CLUSTER UNITS

\begin{tabular}{|c|c|c|c|c|c|c|c|c|}
\hline S.No & Weakness Factors & $\begin{array}{l}\text { Strongly } \\
\text { Agree }\end{array}$ & Agree & Neutral & Disagree & $\begin{array}{l}\text { Strongly } \\
\text { disagree }\end{array}$ & $\begin{array}{l}\text { Total } \\
\text { Score }\end{array}$ & $\begin{array}{l}\text { Mean } \\
\text { Rank }\end{array}$ \\
\hline 1 & High cost of finance & $\begin{array}{c}542 \\
(25.9)\end{array}$ & $\begin{array}{c}552 \\
(26.3)\end{array}$ & $\begin{array}{c}421 \\
(20.1)\end{array}$ & $\begin{array}{c}352 \\
(16.8)\end{array}$ & $\begin{array}{c}224 \\
(10.7) \\
\end{array}$ & 2091 & $\mathrm{X}$ \\
\hline 2 & $\begin{array}{l}\text { Fluctuating price of } \\
\text { raw material }\end{array}$ & $\begin{array}{c}526 \\
(24.9)\end{array}$ & $\begin{array}{c}554 \\
(26.3)\end{array}$ & $\begin{array}{c}446 \\
(21.1)\end{array}$ & $\begin{array}{c}354 \\
(16.8)\end{array}$ & $\begin{array}{c}226 \\
(10.7)\end{array}$ & 2106 & IX \\
\hline 3 & $\begin{array}{l}\text { Inappropriate } \\
\text { infrastructure facility } \\
\text { (Road) }\end{array}$ & $\begin{array}{c}558 \\
(26.3)\end{array}$ & $\begin{array}{c}552 \\
(26.0)\end{array}$ & $\begin{array}{c}458 \\
(21.6)\end{array}$ & $\begin{array}{c}321 \\
(15.1)\end{array}$ & $\begin{array}{c}228 \\
(10.7)\end{array}$ & 2117 & VIII \\
\hline 4 & $\begin{array}{l}\text { Inappropriate } \\
\text { infrastructure facility } \\
\text { (Communication) }\end{array}$ & $\begin{array}{c}635 \\
(34.9)\end{array}$ & $\begin{array}{c}548 \\
(30.1)\end{array}$ & $\begin{array}{c}41 \\
(2.2)\end{array}$ & $\begin{array}{c}365 \\
(20.1)\end{array}$ & $\begin{array}{c}226 \\
(12.4)\end{array}$ & 1815 & XV \\
\hline 5 & $\begin{array}{l}\text { Inappropriate } \\
\text { infrastructure facility } \\
\text { (Water) }\end{array}$ & $\begin{array}{c}621 \\
(28.2)\end{array}$ & $\begin{array}{c}548 \\
(24.9)\end{array}$ & $\begin{array}{l}412 \\
(2.2)\end{array}$ & $\begin{array}{c}366 \\
(16.6)\end{array}$ & $\begin{array}{c}248 \\
(11.2)\end{array}$ & 2195 & V \\
\hline 6 & $\begin{array}{l}\text { Inappropriate } \\
\text { infrastructure facility } \\
\text { (Energy), }\end{array}$ & $\begin{array}{c}531 \\
(25.4)\end{array}$ & $\begin{array}{c}521 \\
(24.9)\end{array}$ & $\begin{array}{c}436 \\
(18.7)\end{array}$ & $\begin{array}{c}354 \\
(16.9)\end{array}$ & $\begin{array}{c}248 \\
(11.8)\end{array}$ & 2090 & XI \\
\hline 7 & $\begin{array}{l}\text { Inappropriate } \\
\text { infrastructure facility } \\
\text { (Logistics) }\end{array}$ & $\begin{array}{c}594 \\
(27.1)\end{array}$ & $\begin{array}{c}526 \\
(24.0)\end{array}$ & $\begin{array}{c}435 \\
(20.8)\end{array}$ & $\begin{array}{c}384 \\
(17.5)\end{array}$ & $\begin{array}{c}246 \\
(11.25)\end{array}$ & 2185 & VI \\
\hline
\end{tabular}


Cluster units in export garment industry: SWOT Analysis

\begin{tabular}{|c|l|c|c|c|c|c|c|c|}
\hline 8 & $\begin{array}{l}\text { Small scale nature of } \\
\text { industry }\end{array}$ & $\begin{array}{c}621 \\
(28.2)\end{array}$ & $\begin{array}{c}524 \\
(23.7)\end{array}$ & $\begin{array}{c}425 \\
(19.3)\end{array}$ & $\begin{array}{c}348 \\
(15.8)\end{array}$ & $\begin{array}{c}284 \\
(12.8)\end{array}$ & 2202 & IV \\
\hline \multirow{2}{*}{9} & Obsolete Technology & $\begin{array}{c}638 \\
(28.6)\end{array}$ & $\begin{array}{c}554 \\
(24.9)\end{array}$ & $\begin{array}{c}422 \\
(18.9)\end{array}$ & $\begin{array}{c}364 \\
(16.3)\end{array}$ & $\begin{array}{c}246 \\
(11.0)\end{array}$ & 2224 & III \\
\hline \multirow{2}{*}{10} & Absence of branding & $\begin{array}{c}548 \\
(25.2)\end{array}$ & $\begin{array}{c}551 \\
(25.3)\end{array}$ & $\begin{array}{c}447 \\
(20.5)\end{array}$ & $\begin{array}{c}354 \\
(16.2)\end{array}$ & $\begin{array}{c}274 \\
(12.6)\end{array}$ & 2174 & VII \\
\hline \multirow{2}{*}{11} & No incentive & $\begin{array}{c}584 \\
(25.6)\end{array}$ & $\begin{array}{c}553 \\
(24.2)\end{array}$ & $\begin{array}{c}446 \\
(19.5)\end{array}$ & $\begin{array}{c}332 \\
(14.5)\end{array}$ & $\begin{array}{c}365 \\
(16.00)\end{array}$ & 2280 & II \\
\hline \multirow{2}{*}{12} & $\begin{array}{l}\text { Pollution control } \\
\text { measures taken by } \\
\text { government }\end{array}$ & $\begin{array}{c}594 \\
(25.6)\end{array}$ & $\begin{array}{c}554 \\
(23.9)\end{array}$ & $\begin{array}{c}432 \\
(18.6)\end{array}$ & $\begin{array}{c}451 \\
(19.4)\end{array}$ & $\begin{array}{c}285 \\
(12.3)\end{array}$ & 2316 & I \\
\hline \multirow{2}{*}{13} & $\begin{array}{l}\text { Lack of } \\
\text { professionalism }\end{array}$ & $\begin{array}{c}555 \\
(28.4)\end{array}$ & $\begin{array}{c}456 \\
(23.3)\end{array}$ & $\begin{array}{c}451 \\
(23.1)\end{array}$ & $\begin{array}{c}225 \\
(11.5)\end{array}$ & $\begin{array}{c}264 \\
(13.5)\end{array}$ & 1951 & XII \\
\hline \multirow{2}{*}{14} & $\begin{array}{l}\text { System of production } \\
\text { contributes to the } \\
\text { delay in delivery }\end{array}$ & $\begin{array}{c}548 \\
(28.1)\end{array}$ & $\begin{array}{c}454 \\
(23.3)\end{array}$ & $\begin{array}{c}432 \\
(22.9)\end{array}$ & $\begin{array}{c}245 \\
(12.6)\end{array}$ & $\begin{array}{c}265 \\
(13.6)\end{array}$ & 1944 & XIII \\
\hline \multirow{2}{*}{16} & $\begin{array}{l}\text { Less investment in } \\
\text { technology up }\end{array}$ & $\begin{array}{c}458 \\
\text { gradation }\end{array}$ & $\begin{array}{c}462 \\
(24.3)\end{array}$ & $\begin{array}{c}432 \\
(22.9)\end{array}$ & $\begin{array}{c}284 \\
(15.07)\end{array}$ & $\begin{array}{c}248 \\
(13.1)\end{array}$ & 1884 & XIV \\
\hline & Delay in Delivery & $\begin{array}{c}456 \\
(29.6)\end{array}$ & $\begin{array}{c}441 \\
(28.6)\end{array}$ & $\begin{array}{c}121 \\
(7.85)\end{array}$ & $\begin{array}{c}254 \\
(16.4)\end{array}$ & $\begin{array}{c}268 \\
(17.4)\end{array}$ & 1540 & XVI \\
\hline
\end{tabular}

When analyzing the various weakness factors, the I rank was secured by the factor X12- Pollution control taken by the government with a total score of 2316, the II, III, ranks were secured by the factors of X11No incentive, X9- Obsolete technology with a total score of 2280, 2224 respectively. The IV, V, VI ranks were secured by the factors of X8- Small scale nature of industry, X5- Inappropriate infrastructure facility,(Water), X7- Inappropriate infrastructure facility ( Logistics ) with a total score of 2202, 2195, 2185 respectively. The VII, VIII, IX and X ranks were secured by the factors of X10- Absence of Branding, X3- Inappropriate infrastructure facility (road) X2- Fluctuating price of raw material , X1- High cost of finance with a total score of 2174, 2117, 2106, 2091 respectively. The XI, XII, XIII, XIV ranks were secured by the factors, $\quad$ X6 Inappropriate infrastructure facility (Energy), X-13 Lack of professionalism, X-14 System of production contributes to the delay in delivery, X-15 Less investment in technology up gradation respectively with a total score of 2090, 1951, 1944, 1884 respectively. The XV, XVI ranks were secured by the factors X-4 inappropriate infrastructure facility (Communication), X-16 Delay in Delivery with a total score of 1815,1540 respectively.

\section{SWOT Analysis - Weakness}

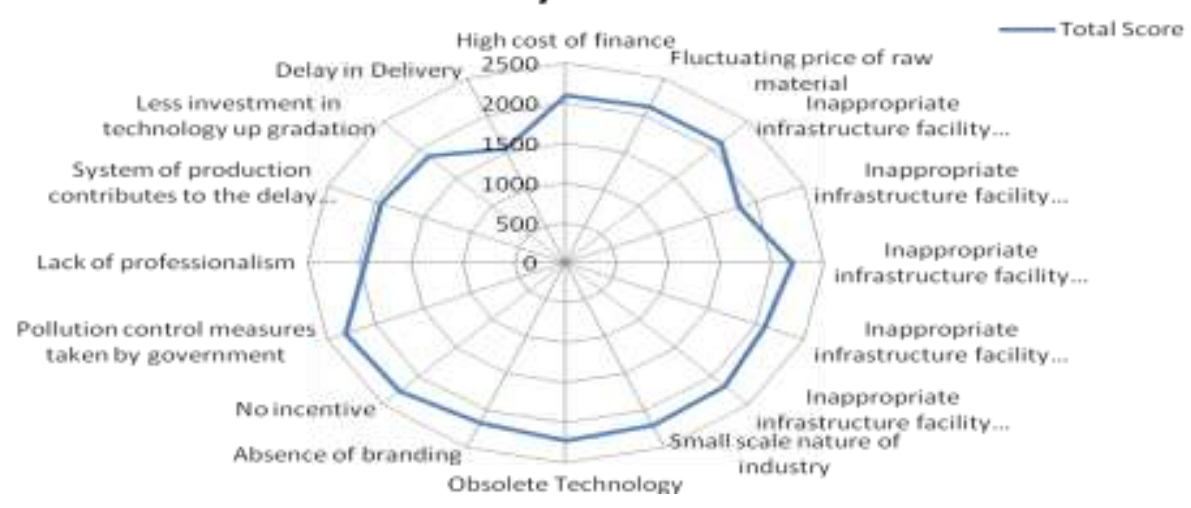

Weakness

Model Summary

\begin{tabular}{|l|r|r|r|r|}
\hline Model & R & R Square & \multicolumn{1}{c|}{$\begin{array}{c}\text { Adjusted R } \\
\text { Square }\end{array}$} & $\begin{array}{c}\text { Std. Error of the } \\
\text { Estimate }\end{array}$ \\
\hline 1 & $.221(\mathrm{a})$ & .049 & .016 & 1.08732 \\
\hline
\end{tabular}

a Predictors: (Constant), delay in delivery, obsolete technology, lack of professionalism, fluctuating price of raw materials, pollution control measures by govt, energy, system of production contributes to the delay 
Cluster units in export garment industry: SWOT Analysis

in delivery, water, small scale nature of industry, absence of branding, communication, less investment in technology upgradation, road, no incentive, high cost of finance, logistics

$\operatorname{ANOVA}(\mathbf{b})$

\begin{tabular}{|l|l|r|r|r|r|r|}
\hline \multirow{2}{*}{ Model } & & $\begin{array}{c}\text { Sum of } \\
\text { Squares }\end{array}$ & \multicolumn{1}{c|}{ df } & Mean Square & \multicolumn{1}{c|}{ F } & \multicolumn{1}{c|}{ Sig. } \\
\hline \multirow{3}{*}{1} & Regression & 28.047 & 16 & 1.753 & 1.483 & $.101(\mathrm{a})$ \\
\cline { 2 - 7 } & Residual & 543.840 & 460 & 1.182 & & \\
\cline { 2 - 8 } & Total & 571.887 & 476 & & & \\
\hline
\end{tabular}

a Predictors: (Constant), delay in delivery, obsolete technology, lack of professionalism, fluctuating price of raw materials, pollution control measures by govt, energy, system of production contributes to the delay in delivery, water, small scale nature of industry, absence of branding, communication, less investment in technology upgradation, road, no incentive, high cost of finance, logistics

b Dependent Variable: form of organization

\begin{tabular}{|c|c|c|c|c|c|c|}
\hline \multicolumn{7}{|c|}{ Coefficients(a) } \\
\hline \multirow{2}{*}{ Model } & & \multicolumn{2}{|c|}{$\begin{array}{l}\text { Unstandardized } \\
\text { Coefficients }\end{array}$} & \multirow{2}{*}{$\begin{array}{c}\begin{array}{c}\text { Standardized } \\
\text { Coefficients }\end{array} \\
\text { Beta }\end{array}$} & \multirow{2}{*}{$\mathbf{t}$} & \multirow{2}{*}{ Sig. } \\
\hline & & B & $\begin{array}{l}\text { Std. } \\
\text { Error }\end{array}$ & & & \\
\hline \multirow[t]{17}{*}{1} & (Constant) & 2.650 & .124 & & 21.431 & .000 \\
\hline & high cost of finance & -.452 & .272 & -.547 & -1.659 & .098 \\
\hline & $\begin{array}{l}\text { fluctuating price of raw } \\
\text { materials }\end{array}$ & -.182 & 183 & -.224 & -.993 & .321 \\
\hline & road & -.313 & .259 & -.376 & -1.211 & .227 \\
\hline & communication & -.137 & .211 & -.168 & -.646 & .519 \\
\hline & water & .074 & .201 & .090 & .367 & .714 \\
\hline & energy & $\begin{array}{ll}-092 \\
\end{array}$ & .160 & -.112 & -.575 & .566 \\
\hline & logistics & .161 & .330 & .197 & .487 & .626 \\
\hline & $\begin{array}{l}\text { small scale nature of } \\
\text { industry }\end{array}$ & -.056 & .185 & -.068 & -.301 & .764 \\
\hline & obsolete technology & .285 & .173 & .349 & 1.652 & .099 \\
\hline & absence of branding & .181 & .223 & .220 & .809 & .419 \\
\hline & no incentive & .152 & .252 & .187 & .602 & .547 \\
\hline & $\begin{array}{l}\text { pollution control measures } \\
\text { by govt }\end{array}$ & -.256 & 185 & -.314 & -1.389 & 166 \\
\hline & lack of professionalism & .113 & .118 & .140 & .955 & .340 \\
\hline & $\begin{array}{l}\text { system of production } \\
\text { contributes to the delay in } \\
\text { delivery }\end{array}$ & -.022 & 146 & -.028 & -.154 & .877 \\
\hline & $\begin{array}{l}\text { less investment in } \\
\text { technology upgradation }\end{array}$ & .593 & .216 & .722 & 2.739 & .006 \\
\hline & delay in delivery & -.118 & .112 & -.144 & -1.050 & .294 \\
\hline
\end{tabular}

a Dependent Variable: form of organisation

When the weakness factor was analysed with the multiple regression technique it was under stood that all the selected factors were having significance with the form of organization where the job work is undertaken. 


\section{TABLE-4}

LEVEL OF AGREEMENT ON THE VARIOUS OPPORTUNITY FACTORS OF THE PERFORMANCE OF CLUSTER UNITS

\begin{tabular}{|c|l|c|c|c|c|c|c|c|}
\hline S.No & Opportunity Factors & $\begin{array}{c}\text { Strongly } \\
\text { Agree }\end{array}$ & Agree & Neutral & Disagree & $\begin{array}{c}\text { Strongly } \\
\text { Disagree }\end{array}$ & $\begin{array}{c}\text { Total } \\
\text { score }\end{array}$ & $\begin{array}{c}\text { Mean } \\
\text { rank }\end{array}$ \\
\hline \multirow{2}{*}{1} & $\begin{array}{l}\text { Removal of quota } \\
\text { restriction under WTO } \\
\text { with effect } \\
\text { from1.1.2005 }\end{array}$ & $\begin{array}{c}654 \\
(25.08)\end{array}$ & $\begin{array}{c}548 \\
(21.0)\end{array}$ & $\begin{array}{c}564 \\
(21.6)\end{array}$ & $\begin{array}{c}354 \\
(13.5)\end{array}$ & $\begin{array}{c}487 \\
(18.6)\end{array}$ & 2607 & V \\
\hline \multirow{2}{*}{2} & $\begin{array}{l}\text { Falling share of } \\
\text { garment manufacture } \\
\text { of other countries. }\end{array}$ & $\begin{array}{c}625 \\
(23.3)\end{array}$ & $\begin{array}{c}652 \\
(24.3)\end{array}$ & $\begin{array}{c}541 \\
(20.2)\end{array}$ & $\begin{array}{c}362 \\
(13.5)\end{array}$ & $\begin{array}{c}495 \\
(18.5)\end{array}$ & 2675 & II \\
\hline 3 & $\begin{array}{l}\text { Preference of Indian } \\
\text { garments over others. }\end{array}$ & $\begin{array}{c}631 \\
(24.1)\end{array}$ & $\begin{array}{c}532 \\
(20.3)\end{array}$ & $\begin{array}{c}532 \\
(20.3)\end{array}$ & $\begin{array}{c}458 \\
(17.5)\end{array}$ & $\begin{array}{c}457 \\
(17.5)\end{array}$ & 2610 & IV \\
\hline \multirow{2}{*}{5} & $\begin{array}{l}\text { Cluster approach } \\
\text { gaining momentum }\end{array}$ & $\begin{array}{c}668 \\
(24.88)\end{array}$ & $\begin{array}{c}551 \\
(20.5)\end{array}$ & $\begin{array}{c}551 \\
(20.5)\end{array}$ & $\begin{array}{c}465 \\
(17.3)\end{array}$ & $\begin{array}{c}449 \\
(16.7)\end{array}$ & 2684 & I \\
\hline \multirow{2}{*}{6} & Trade fairs & $\begin{array}{c}658 \\
(29.3)\end{array}$ & $\begin{array}{c}556 \\
(21.1)\end{array}$ & $\begin{array}{c}501 \\
(19.0)\end{array}$ & $\begin{array}{c}447 \\
(17.9)\end{array}$ & $\begin{array}{c}46.3 \\
(17.6)\end{array}$ & 2625 & III \\
\hline
\end{tabular}

When the Opportunity factors were analysed with the various factors which are taken in to consideration, the I rank was secured by the factor of X4- Cluster approach gaining momentum, with a total score of 2684, the II and III franks were gained by the factors of X2- Falling share of garment manufacturers of other countries, X-5 Trade fairs with a total score of 2675, 2625 respectively. The IV, V, VI ranks were secured by the factors of X3- Preference of Indian garment over others, X1- Removal of quota under WTO with effect from 1.1.2005 X6- Strength of trade association respectively with a total score of 2610, 2607, 2257 respectively.

\section{SWOT Analysis - Opportunity}

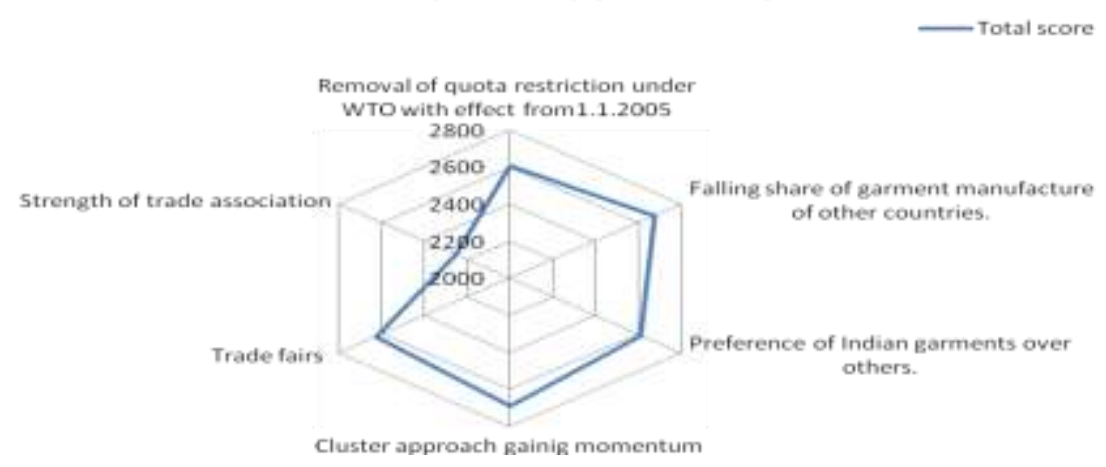

Opportunity

Model Summary

\begin{tabular}{|l|r|r|r|r|}
\hline Model & R & R Square & \multicolumn{1}{c|}{$\begin{array}{c}\text { Adjusted R } \\
\text { Square }\end{array}$} & $\begin{array}{c}\text { Std. Error of the } \\
\text { Estimate }\end{array}$ \\
\hline 1 & $.166(\mathrm{a})$ & .028 & .015 & 1.08776 \\
\hline
\end{tabular}

a Predictors: (Constant), strength of trade associations, removal of quota restriction under WTO with effect, falling share of garment manufacture of other countries, preference of Indian garments over others, cluster approach gaining momentum, trade fairs 
Cluster units in export garment industry: SWOT Analysis ANOVA(b)

\begin{tabular}{|l|l|r|r|r|r|c|}
\hline \multirow{2}{*}{ Model } & & $\begin{array}{c}\text { Sum of } \\
\text { Squares }\end{array}$ & \multicolumn{1}{c|}{ df } & $\begin{array}{c}\text { Mean } \\
\text { Square }\end{array}$ & F & Sig. \\
\hline \multirow{3}{*}{1} & Regression & 15.775 & 6 & 2.629 & 2.222 & $.040(\mathrm{a})$ \\
\cline { 2 - 8 } & Residual & 556.112 & 470 & 1.183 & & \\
\cline { 2 - 8 } & Total & 571.887 & 476 & & & \\
\hline
\end{tabular}

a Predictors: (Constant), strength of trade associations, removal of quota restriction under WTO with effect, falling share of garment manufacture of other countries, preference of Indian garments over others, cluster approach gaining momentum, trade fairs b Dependent Variable: form of organisation

\begin{tabular}{|c|c|c|c|c|c|c|}
\hline \multicolumn{7}{|c|}{ Coefficients(a) } \\
\hline \multirow[t]{2}{*}{ Model } & & \multicolumn{2}{|c|}{$\begin{array}{l}\text { Unstandardized } \\
\text { Coefficients }\end{array}$} & \multirow{2}{*}{$\begin{array}{c}\begin{array}{c}\text { Standardize } \\
\text { d } \\
\text { Coefficients }\end{array} \\
\text { Beta }\end{array}$} & \multirow[t]{2}{*}{$\mathbf{t}$} & \multirow[t]{2}{*}{ Sig. } \\
\hline & & B & $\begin{array}{c}\text { Std. } \\
\text { Error }\end{array}$ & & & \\
\hline \multirow[t]{7}{*}{1} & (Constant) & 2.571 & .120 & & 21.351 & .000 \\
\hline & $\begin{array}{l}\text { removal of quota } \\
\text { restriction under } \\
\text { WTO with effect }\end{array}$ & .196 & .148 & .240 & 1.319 & .188 \\
\hline & $\begin{array}{l}\text { falling share of } \\
\text { garment manufacture } \\
\text { of other countries }\end{array}$ & -.129 & .220 & -.159 & -.584 & .559 \\
\hline & $\begin{array}{l}\text { preference of Indian } \\
\text { garments over others }\end{array}$ & -.476 & .235 & -.581 & -2.027 & .043 \\
\hline & $\begin{array}{l}\text { cluster approach } \\
\text { gaining momentum }\end{array}$ & .629 & .252 & .766 & 2.501 & .013 \\
\hline & trade fairs & -.317 & .304 & -.389 & -1.040 & .299 \\
\hline & $\begin{array}{l}\text { strength of trade } \\
\text { associations }\end{array}$ & .050 & .124 & .062 & .403 & .687 \\
\hline
\end{tabular}

a Dependent Variable: form of organisation

When the opportunity factor was analyzed with the multiple regression technique it was under stood that all the selected factors were having significance with the form of organization where the job work is undertaken.

TABLE-5

LEVEL OF AGREEMENT ON THE VARIOUS THREAT FACTORS OF THE PERFORMANCE OF CLUSTER UNITS

\begin{tabular}{|c|l|c|c|c|c|c|c|c|}
\hline S.No & \multicolumn{1}{|c|}{ Treat Factors } & $\begin{array}{c}\text { Strongly } \\
\text { Agree }\end{array}$ & Agree & Neutral & Disagree & $\begin{array}{c}\text { Strongly } \\
\text { disagree }\end{array}$ & $\begin{array}{c}\text { Total } \\
\text { Score }\end{array}$ & $\begin{array}{c}\text { Mean } \\
\text { Rank }\end{array}$ \\
\hline 1 & $\begin{array}{l}\text { Phasing out of } \\
\text { quantitative restriction } \\
\text { of quota }\end{array}$ & $\begin{array}{c}487 \\
(24.01)\end{array}$ & $\begin{array}{c}547 \\
(26.9)\end{array}$ & $\begin{array}{c}401 \\
(19.7)\end{array}$ & $\begin{array}{c}365 \\
(17.9)\end{array}$ & $\begin{array}{c}228 \\
(11.2)\end{array}$ & 2028 & VIII \\
\hline 2 & $\begin{array}{l}\text { Emergence of new } \\
\text { production center like } \\
\text { Vietnam, Pakistan, } \\
\text { Srilanka, Bangladesh. }\end{array}$ & $\begin{array}{c}569 \\
(24.8)\end{array}$ & $\begin{array}{c}487 \\
(21.2)\end{array}$ & $\begin{array}{c}584 \\
(25.5)\end{array}$ & $\begin{array}{c}384 \\
(16.7)\end{array}$ & $\begin{array}{c}265 \\
(11.5)\end{array}$ & 2289 & V \\
\hline 3 & Unpopularity & $\begin{array}{c}574 \\
(27.0)\end{array}$ & $\begin{array}{c}436 \\
(20.5)\end{array}$ & $\begin{array}{c}465 \\
(21.7)\end{array}$ & $\begin{array}{c}367 \\
(17.3)\end{array}$ & $\begin{array}{c}278 \\
(13.1)\end{array}$ & 2120 & VII \\
\hline 4 & $\begin{array}{l}\text { Competition with } \\
\text { other firms. }\end{array}$ & $\begin{array}{c}598 \\
(25.4)\end{array}$ & $\begin{array}{c}479 \\
(20.3)\end{array}$ & $\begin{array}{c}447 \\
(18.9)\end{array}$ & $\begin{array}{c}465 \\
(19.7)\end{array}$ & $\begin{array}{c}365 \\
(15.5)\end{array}$ & 2354 & I \\
\hline 5 & $\begin{array}{l}\text { Government policy } \\
\text { changes with regard to } \\
\text { export. }\end{array}$ & $\begin{array}{c}546 \\
(23.8)\end{array}$ & $\begin{array}{c}489 \\
(21.3)\end{array}$ & $\begin{array}{c}468 \\
(20.4)\end{array}$ & $\begin{array}{c}443 \\
(19.3)\end{array}$ & $\begin{array}{c}345 \\
(15.0)\end{array}$ & 2291 & IV \\
\hline \multirow{2}{*}{6} & Small size of units. & $\begin{array}{c}523 \\
(23.6)\end{array}$ & $\begin{array}{c}548 \\
(24.7)\end{array}$ & $\begin{array}{c}354 \\
(16.01)\end{array}$ & $\begin{array}{c}417 \\
(18.8)\end{array}$ & $\begin{array}{c}368 \\
(16.6)\end{array}$ & 2210 & VI \\
\hline
\end{tabular}


Cluster units in export garment industry: SWOT Analysis

\begin{tabular}{|c|l|c|c|c|c|c|c|c|}
\hline 7 & Pollution problems. & $\begin{array}{c}518 \\
(22.3)\end{array}$ & $\begin{array}{c}568 \\
(24.5)\end{array}$ & $\begin{array}{c}446 \\
(19.2)\end{array}$ & $\begin{array}{c}446 \\
(19.2)\end{array}$ & $\begin{array}{c}339 \\
(14.6)\end{array}$ & 2317 & III \\
\hline 8 & Economic downturn. & $\begin{array}{c}555 \\
(23.6)\end{array}$ & $\begin{array}{c}497 \\
(21.14)\end{array}$ & $\begin{array}{c}432 \\
(18.38)\end{array}$ & $\begin{array}{c}498 \\
(21.19)\end{array}$ & $\begin{array}{c}368 \\
(15.6)\end{array}$ & 2350 & II \\
\hline
\end{tabular}

The above table presents the various threat factors of the job workers in Tirupur city. The I rank was Secured by the factor X4- Competition with other firms, the II, III and IV ranks were secured by the factors of X8- Economic down turn, X7- Pollution problems, X5- Government policy changes with regard to export with a total score of 2350, 2317, 2291 respectively. The V, VI, VII, VIII ranks were observed by the factors of X2Emergence of new production center like Vietnam, Pakistan, Srilanka, Bangladesh., X6- Small size of units, X3- Unpopularity, X1- Phasing out of quantitative restriction of quota with a total score of 2289, 2210, 2120, 2028 respectively.

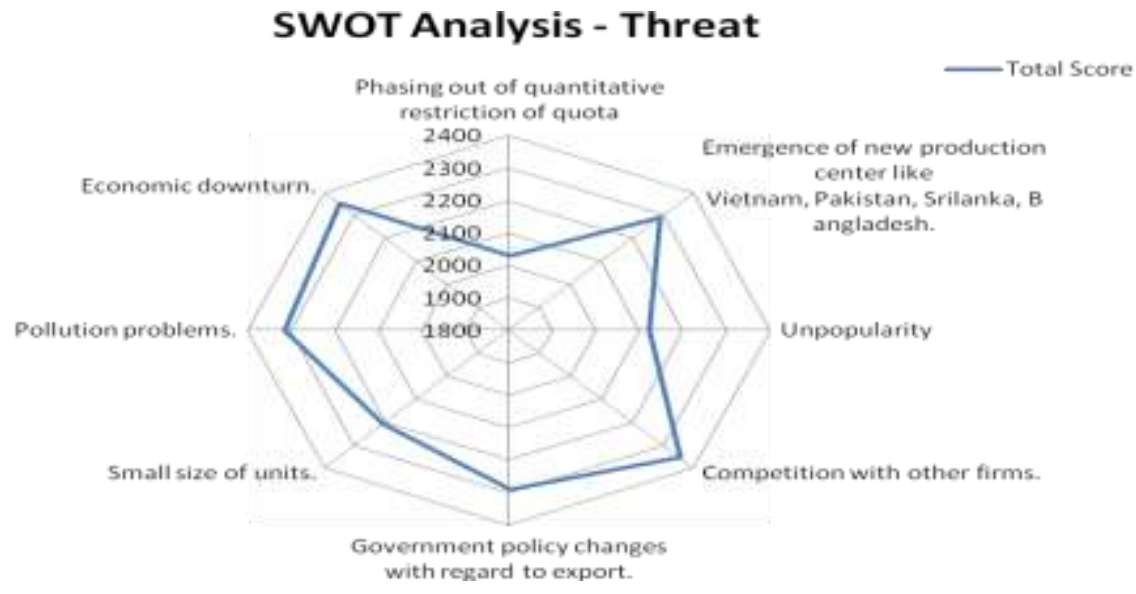

Threats

Model Summary

\begin{tabular}{|c|r|r|r|r|}
\hline Model & R & R Square & \multicolumn{1}{c|}{$\begin{array}{c}\text { Adjusted R } \\
\text { Square }\end{array}$} & $\begin{array}{c}\text { Std. Error of the } \\
\text { Estimate }\end{array}$ \\
\hline 1 & $.126(\mathrm{a})$ & .016 & -.001 & 1.09662 \\
\hline
\end{tabular}

a Predictors: (Constant), economic downturn, govt plicy changes with regard to export, pollution problems, emergence of new production center like vietnam, pakistan, srilanka, bangaladesh, small size of units, unpopularity, phasing out of quantitative restriction of quota, competition with other firms

$\operatorname{ANOVA}(\mathbf{b})$

\begin{tabular}{|l|l|r|r|r|r|r|}
\hline Model & & $\begin{array}{c}\text { Sum of } \\
\text { Squares }\end{array}$ & \multicolumn{1}{c|}{ df } & Mean Square & \multicolumn{1}{c|}{ F } & \multicolumn{1}{c|}{ Sig. } \\
\hline 1 & Regression & 9.086 & 8 & 1.136 & .944 & $.479(\mathrm{a})$ \\
\hline & Residual & 562.801 & 468 & 1.203 & & \\
\hline & Total & 571.887 & 476 & & & \\
\hline
\end{tabular}

a Predictors: (Constant), economic downturn, govt plicy changes with regard to export, pollution problems, emergence of new production center like vietnam, pakistan, srilanka, bangaladesh, small size of units, unpopularity, phasing out of quantitative restriction of quota, competition with other firms b Dependent Variable: form an organization.

\begin{tabular}{|l|l|r|r|r|r|r|}
\hline \multirow{2}{*}{ Model } & & \multicolumn{1}{|c|}{$\begin{array}{c}\text { Coefficients(a) } \\
\text { Coefficients }\end{array}$} & $\begin{array}{c}\text { Standardized } \\
\text { Coefficients }\end{array}$ & \multirow{2}{*}{ T } & \multirow{2}{*}{ Sig. } \\
\hline & & \multicolumn{1}{c|}{ B } & \multicolumn{1}{c|}{$\begin{array}{c}\text { Etd. } \\
\text { Error }\end{array}$} & \multicolumn{1}{c|}{ Beta } & & \\
\hline 1 & (Constant) & 2.581 & .121 & & 21.323 & .000 \\
\hline & $\begin{array}{l}\text { phasing out of quantitative } \\
\text { restriction of quota }\end{array}$ & -.077 & .246 & -.094 & -.311 & .756 \\
\hline
\end{tabular}


Cluster units in export garment industry: SWOT Analysis

\begin{tabular}{|c|c|c|c|c|c|}
\hline $\begin{array}{l}\text { emergence of new production } \\
\text { center like vietnam, pakistan, } \\
\text { srilanka, bangaladesh }\end{array}$ & -.005 & .139 & -.006 & -.036 & .971 \\
\hline unpopularity & -.100 & .219 & -.123 & -.455 & .649 \\
\hline competition with other firms & -.249 & .378 & -.306 & -.658 & .511 \\
\hline $\begin{array}{l}\text { govt plicy changes with regard } \\
\text { to export }\end{array}$ & .243 & .110 & .301 & 2.207 & .028 \\
\hline small size of units & .110 & .178 & .137 & .616 & .538 \\
\hline pollution problems & .019 & .116 & .023 & .167 & .868 \\
\hline economic downturn & .017 & .111 & .022 & .157 & .876 \\
\hline
\end{tabular}

a Dependent Variable: form of organization

When the Threat factor was analyzed with the multiple regression technique it was under stood that all the selected factors were having significance with the form of organization where the job work is undertaken

\section{IV.Conclusion}

Tirupur Garment cluster units has shown a lofty performance over a long period and has been a back bone for the garment industry to contribute a major share to the country's economy and foreign exchange .There are certain core issues that require urgent attention of the government. These issues are Infrastructural bottleneck, pollution control, and obsolete technology. Tirupur has well poised in garment industry because of its various strength, Indian government may come forward to take measures with soaring spirit for the pollution control, and provide incentives and drawback to perk up Tirupur from its crisis.

\section{References}

[1]. Aston S. O., Masselink. H. and. Provost .J. R, Jet printing with reactive dyes, J. Soc.Dyers , Color., 109, 147-152 (1993)

[2]. Cowthrne (1995), Of Networks and Markets: the rise and rise of a South Indian , Town, Tirpur

[3]. David J. Spencer 2001, Textile Research Journal, Volume 81, 38-40.

[4]. Englewood Cliffs N.J 1999, Journal of Fibre \& Textile Research, Vol. 33, 2007

[5]. Jaitley,A.(2003).Exports:asuccessstory.RetrievedNovember25,2005, from http://commerce.nic.in/exports_success.pdf

[6]. Sweet. R. G. Signal apparatus with fluid drop recorder, US Patent 3,596,275 (1971).

[7]. Tea Association Magazine-Bulletin, Feb-2011

[8]. Veembur, A. (2004). Texitile exports: India spins to lead. Siliconindia, 8(2), 44-46.

[9] www.tea-india.org 